\title{
Evaluation of Distance Course Effectiveness - Exploring the Quality of Interactive Processes
}

\author{
Francisco Villa Ulhôa Botelho ${ }^{1}$ and Rosa Maria Vicari ${ }^{2}$ \\ ${ }^{1}$ Universidade Católica de Brasília, UCB, Brazil \\ fbotelho@ucb.br \\ ${ }^{2}$ Universidade Federal do Rio Grande do Sul, UFRGS, Brazil \\ rosa@inf.ufrgs.br
}

\begin{abstract}
Understanding the dynamics of learning processes implies an understanding of their components: individuals, environment or context and mediation. It is known that distance learning (DL) has a distinctive characteristic in relation to the mediation component. Due to the need of overcoming the barriers of distance and time, DL intensively uses information and communication technologies (ICT) to perform interactive processes. Construction of effective learning environments depends on human relationships. It also depends on the emotionality placed on such relationships. Therefore, knowing how to act in virtual environments in the sense of creating the required ambiance for animation of learning processes has a unique importance. This is the theme of this study. Its general objectives were achieved and can be summarized as follows: analyze indexes that are significant for evaluations of distance course effectiveness; investigate to which extent effectiveness of DL courses is correlated with quality of interactive processes; search characteristics of the conversations by individuals interacting in study groups that are formed in virtual environments, which may contribute to effectiveness of distance courses.
\end{abstract}

Keywords: DL quality, evaluation of DL effectiveness, interactive processes in DL, evaluation of DL interactivity.

\section{Theoretical Background for an Evaluation of Distance Course Effectiveness}

Distance learning is an education modality, and education is basically learning. Thus, learning and interactive processes are the most comprehensive themes when thinking about theoretical references that may consolidate our research path.

Two approaches that could complement each other when guiding studies on effectiveness of interactive processes in virtual learning environments are Vygotsky's history and cultural theory [1] and the foundations of complex thinking. The rationale for choosing these theoretical approaches is the understanding that learning should be evaluated without being reduced to the result of a formal teaching action, in which the individual is passive and the phenomenon can be isolated from its context. Learning, on the other hand, should be treated as the action of people interacting with other 
people and with their environment in the process of production, conservation and change in their lives. In such case, the phenomenon comprehends several interacting elements, conforming a complex reality.

Vygotsky's history and cultural approach $[1,2,3]$ was used as an attempt to understand the importance of interactive processes for human learning: how people learn and develop themselves. Complex thinking served to provide the orientation that learning does not obey a linear cause-effect logic, but is a dynamic process, as a result and factor of multiple interactions, as part of the self-organization process of people and society.

Both approaches provided a definition of learning and understanding of its process. Theoretical references were used to aid the process of effectiveness evaluation in DL courses. Therefore, these approaches provided teachings that not only indicate components of the human learning system to be evaluated, but also their interconnections and movements, their self-organization.

The phenomenon of human interactions focused on formal educational processes represents a complex system, which establishes relationships with their social and historic context. Education is, before any thing, a dynamic process in which many elements - student, teacher, mediation instruments and signs, environment - are interacting so that results are not necessarily predictable, do not obey a simple cause/effect process.

We are convinced that the learning phenomenon and its effects in students' practices cannot be reduced to a product or result of educational actions. Treating such a rich phenomenon as a result of a mere sum of actions is closing the door to an understanding of its totality. Without discarding the necessary educational planning, one should be open to aspects of an emerging nature that every educational process has.

\section{Description of a Possible Path for Evaluation of Distance Course Effectiveness}

\subsection{Characteristics of the Study Case}

One of the main difficulties of studies on evaluation of effectiveness of courses or educational programs is the follow-up of all stages, that is, action onset, process, end and subsequent step, in which results are analyzed. DL actions usually have some components that make evaluation even harder, such as space comprehensiveness of the action, quantity and heterogeneity of the target audience. Such difficulties indicate a possible justification for the lack of studies on DL effectiveness.

As an attempt to overcome the challenges of studying DL effectiveness, an ongoing DL experience was chosen as the object of this study. It is a course that, since it was implemented, has been planned having an evaluation project as a component. Such course, as well as data collection instruments, were developed by the team of Universidade Católica de Brasília Virtual (UCB VIRTUAL).

It is a formation process for higher education teachers with the aim of improving the quality of teaching-learning practices. This course is developed at a virtual environment with some in-person meetings. Its didactic material is structured in 
Autonomous Study Units (ASU). Each ASU is comprised of contents as hypertexts, case studies and exercises, references and website, conventional digitized texts for complementary reading.

The entire follow-up of the learning process is performed by teachers through a tutoring system composed of two in-person meetings and interactive processes performed at a virtual environment. The most widely used interactive tools are chats and discussion forums.

\subsection{Indexes of Results/Impact of Distance Courses}

The main sources of research information are apprentices (higher education teachers), their colleagues, coordinators of the areas in which apprentices act, and students belonging to classes of courses taught in their respective institutions.

Information is collected by electronic questionnaires using close-ended and openended questions. Data collection process is performed according to the model described in Figure 1.

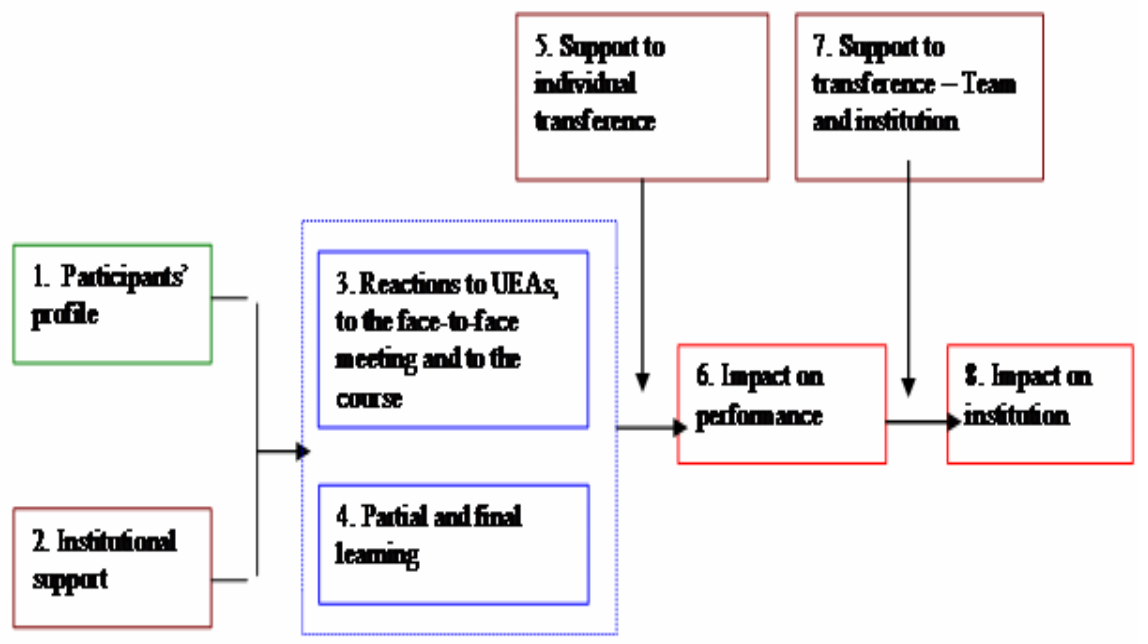

\section{At the conse start During the canrse After the course}

Fig. 1. Evaluation model (based on the model of five stages developed by Abbad (2000))

This model is an evolution of the traditional approach by Kirkpatrick (1976) and Hamblin (1978), both cited in [4]. Such a model enables collection of information for studies at the level of students' reaction to formation, learning, students' behavior at work or formation impact, organizational change, and final value measured to improve the quality of products and services within organizations (the last three levels are performed in formations offered based on a given institutional demand). 
Although the analysis process uses nearly all information collected according to the described model, indexes focused by his study are those relative to results. Such indexes are comprised of the information described in item 6 of Figure 1 above, namely, change in professional performance as a consequence of the course (changes in aspects regarding course objectives or deep impact).

Evaluation questionnaires contain questions relating to impact, to try and capture changes in participants' performance - in their teaching activity - in relation to course objectives. The items included in the questionnaire completed by course participants have a correspondence in the questions completed by their students, regularly enrolled in one of the courses taught in their institution.

\subsection{Indexes of Interactive Process Quality}

Human learning has multiple dimensions: from psychosocial to historic. Such dimensions turn it into a complex phenomenon. To study it from the complex thinking perspective, it was necessary to have a methodology that could guide analysis of interactive processes in a virtual environment. The Meta Learning model proposed by Losada[5] was chosen.

The Meta Learning model proposes a view on the learning process of people interacting in a group. It is an explanatory model of the functioning dynamics of teams and of the interaction between people that compose such teams. It is focused on the understanding of factors contributing to creation and maintenance of high performance group dynamics.

After testing and analyzing a set of variables representative of "speeches" of people in conversation processes, Losada's study reached three bipolar variables that can be extremely significant to explain interaction dynamics. Depending on such dynamics, groups have a performance marked by creativity and innovation or by common sense and repetition. They are descriptive variables of people's postures in interactive processes. They indicate the quality of people's attitudes toward the "speeches" of others in relation to the characteristics of positivity or negativity, questioning or proposition, being focused on oneself or on the other (Table 1).

Table 1. Matrix of team performance [6]

\begin{tabular}{|c|c|c|c|c|c|}
\hline \multirow[t]{2}{*}{ Performance } & \multirow[t]{2}{*}{ Dynamics } & \multirow[t]{2}{*}{ Connectivity } & \multicolumn{3}{|c|}{ Bipolar Variables } \\
\hline & & & $\begin{array}{l}\text { Questioning/ } \\
\text { Proposition }\end{array}$ & $\begin{array}{l}\text { The other/ } \\
\text { Oneself }\end{array}$ & $\begin{array}{l}\text { Positivity/ } \\
\text { Negativity } \\
\text { (Emotional } \\
\text { Space) }\end{array}$ \\
\hline High & Complexor & High & Balanced & Balanced & $\begin{array}{l}\text { Expansive } \\
\mathrm{P} \gg \mathrm{N}\end{array}$ \\
\hline Medium & Limit cycle & Medium & $\begin{array}{l}\text { Tendency to } \\
\text { unbalance in the } \\
\text { direction of } \\
\text { proposition }\end{array}$ & $\begin{array}{l}\text { Unbalanced } \\
\text { oriented to oneself }\end{array}$ & $\begin{array}{l}\text { Restrictive } \\
\mathrm{P}>\mathrm{N}\end{array}$ \\
\hline Low & Fixed point & Low & $\begin{array}{l}\text { Unbalanced in } \\
\text { the direction of } \\
\text { proposition }\end{array}$ & $\begin{array}{l}\text { Entirely } \\
\text { unbalanced } \\
\text { oriented to oneself }\end{array}$ & $\begin{array}{l}\text { Highly } \\
\text { restrictive } \\
\mathrm{N}>\mathrm{P}\end{array}$ \\
\hline
\end{tabular}


Among bipolar variables, positivity/negativity is crucial for interactive processes. It operates as a powerful feedback system to generate different emotional spaces. Such spaces are represented in the Meta Learning model (Figure 2) by P/N ratio (total number of positive interventions divided by total number of negative interactions). When this ratio is within an adequate range, people are creating expansive emotional spaces that increase connectivity and cause better group performance. Outside this range means that actions are resulting in restrictive emotional spaces that discourage performance.

$\mathrm{P} / \mathrm{N}$ ratio is calculated and set within a reference range called Losada Line, in which positivity ranges between 2.9013 or more (not higher than 11.6153) to 1 of negativity. This means that, for each negative intervention in team interactions there must be at least about three positive interventions.

Reference in history and cultural approach and in complex thinking is clearly manifested in two expressions: dynamics of interactive processes or conversational dynamics. Analysis of interactive processes as a way to understand the quality of DL courses is supported by Vygotsky's concept of learning. The theoretical references mentioned above also change the focus to dynamics of interactive processes, as they see the phenomenon of human learning as an open system that establishes relationships with their social and historic context.

Therefore, when quality of interactive processes is sought, the answer to the following question is being built: What type of intervention and how interventions are combined in group contexts to generate a positive conversational dynamic for learning and for effectiveness of courses (such dynamic was called "complexor" by Losada)? This is a crucial issue in the development of indexes and to create an analysis able to describe such dynamics and its attractors.

In any type of communication between people it is known that, when certain "speeches" are said, conversation could be terminated or reduced to repetitions, or generate an animation that might ensure that people are open to continuing the conversation or satisfactorily resuming it in another opportunity. This takes place in conversations held by a family group, a group of friends or in a classroom. Teachers know when a class acquires a dynamic of strong participation and interest or when it seems they are talking to the walls. What are the interventions that lead to each of these dynamics?

In the study of discussion forums, two pairs of bipolar variables presented in the Meta Learning model by Losada \& Heaphy [6] were adopted. Such adaptation was performed by suppressing one of the three pairs of variables from the model and rewriting the description of two other pairs.

The need for eliminating one pair of variables is justified by the fact that Losada's study was performed by observation of people/teams during in-person conservations, so that body manifestation of participants in conversations (the body talks) was also observed and information was added to capture of variables. In our case, variables were captured from records in discussion forums; therefore, a written verbal communication, which prevents observation of participants' body aspects. 


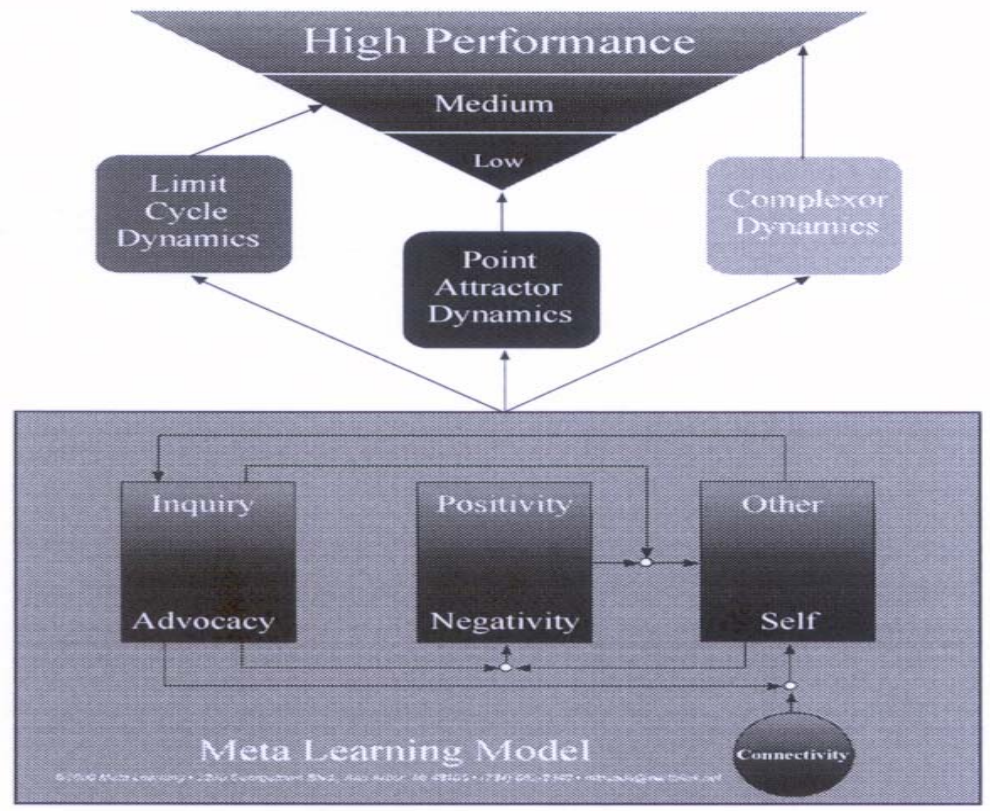

Fig. 2. Losada's Meta Learning model [6]

Table 2. Definition of variables to analyze interactive processes

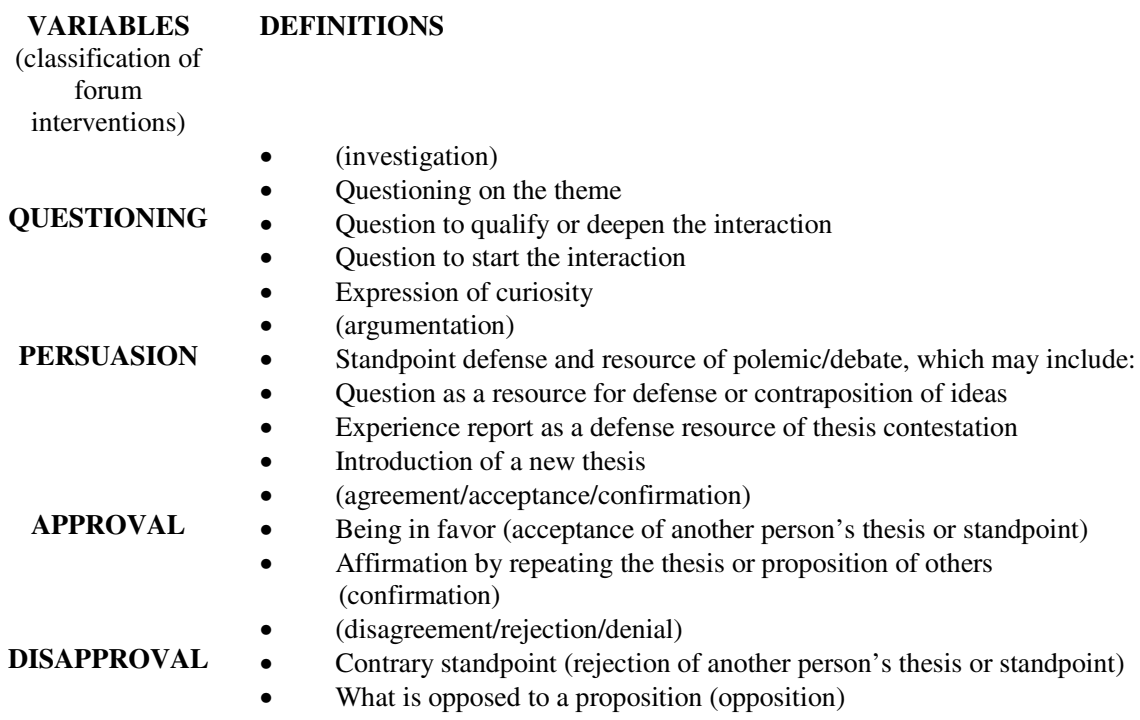

As can be seen in Table 2, the variables questioning/proposition and positivity/ negativity were redefined in the Meta Learning model. The former was replaced by questioning/persuasion, and the latter by approval/disapproval. 


\subsection{Procedures for Analysis of the Correlation between Impact Results and Characteristics of Interactive Processes}

The procedure for analysis preparation consisted of comparing the results of two analytic processes, structured based on the five-stage model and on the adapted Meta Learning model.

From the five-stage model (see Figure 1) basically the results of impact evaluation were used. The Meta Learning model was used to develop an adaptation for a better codification of written verbal communication: forums were codified and analyzed based on the bipolar variables questioning/persuasion and agreement/disagreement (see Table 2). Such variables use those applied in the Meta Learning model as reference; however, their content was redefined for a better standardization of text codification in forums, as shown in Figure 3.

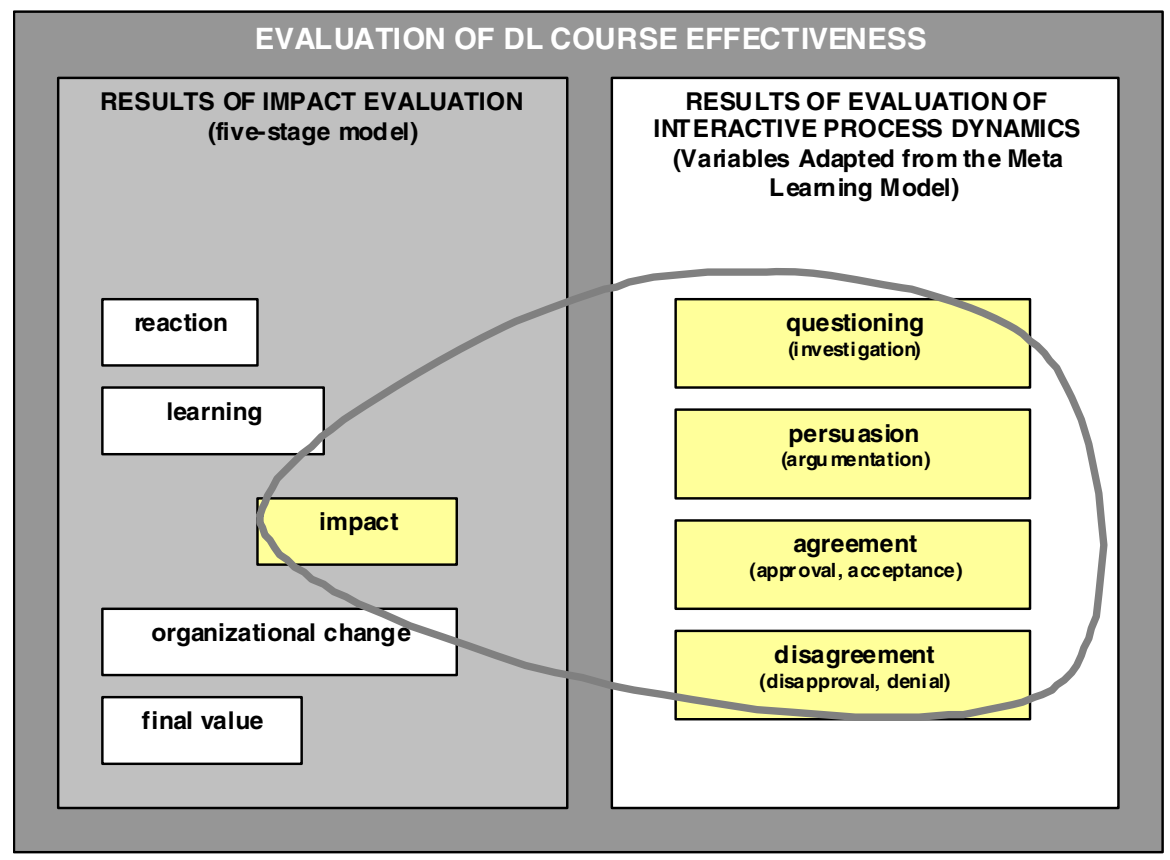

Fig. 3. Model of effectiveness evaluation applied in this study

Analysis of forums, with results expressing incidence of each variable in students' and tutors' "speeches" or interventions, was compared with mean scores of impact evaluation obtained by students. Thus, it was possible to obtain the characteristics of actors' "speeches" in forums according to groups with larger concentration of high or low impact evaluations.

For the same groups, classified according to quality in impact evaluation, forum dynamics was observed by analysis of the evolution of "speech" configurations from the first to the last forums throughout the course. 
In summary, the script for the process of information analysis went through the following steps:

a) Searching, using impact indexes, people with the highest or lowest evaluations;

b) Verifying in which groups/classes such people are and whether there is a hegemony in relation to impact evaluation, i.e., relative to existence or not of groups with concentration of people with high or low impact evaluation.

In case groups of high or low impact were observed:

a) Identifying distinctive conversation characteristics in discussion forums using two bipolar variables in the adapted Meta Learning model, as well as distinctive characteristics associated with the quantitative of interventions;

b) Verifying the evolution (dynamics) of such conversational characteristics, comparing results between the first and last forums.

The following resources of descriptive statistics were used to analyze information and summarize results:

a) Descriptive graphs and tables;

b) Parametric description, in which the values of certain parameters were estimated to facilitate description of the data set: measures of central tendency and measures of statistical dispersion;

c) Correlations between variables.

Understanding that statistical results are only a support for the understanding of phenomena guided the analysis. Although correlations between many variables have been entirely explored, only those that contained significant information for an understanding of research questions were used.

\section{Summary of Results}

Understanding the dynamics of learning processes implies an understanding of its components: individuals, environment or context and mediation. It is known that distance learning (DL) has a distinctive characteristic in relation to the mediation component. Due to the need of overcoming the barriers of distance and time, DL intensively uses information and communication technologies (ICT) to perform interactive processes.

Construction of effective learning environments depends on human relationships. It also depends on the emotionality placed on such relationships. Therefore, knowing how to act in virtual environments in the sense of creating the required ambiance for animation of learning processes has a unique importance.

The general objectives of this study were reached: analyze indexes that are significant for evaluations of distance course effectiveness; investigate to which extent effectiveness of DL courses is correlated with quality of interactive processes; search characteristics of the conversations of individuals interacting in study groups that are formed in virtual environments, which may contribute to effectiveness of distance courses. 
The main results of the analysis corroborate such statements, and can be summarized as follows:

a) There was a positive evaluation of the course by participants;

b) Final evaluation scores of course participants and their self-evaluations after ending the course were not good indexes of course effectiveness; results indicated inexistence of a correlation between final score and self-evaluation and impact evaluation;

c) Context proved to be important for course effectiveness;

d) Group and interaction dynamics between its components were considered as important for course effectiveness;

e) Teacher's activity was also considered as relevant for course effectiveness; his presence, with constant interventions throughout the entire course (initial and final forums), is significant for group impact; quality and balance (bipolar variables) of the teacher's interventions throughout the course are significant for group impact;

f) As to characteristics of interventions in the internal conversation of learning groups, persuasion stood out as a marked characteristic in conversations of both groups (high and low impact); there was an unbalance in the direction of persuasion in the bipolar variable questioning/persuasion in both groups; only high-impact groups had a compatibility between approval/disapproval variables and Losada Line (high-impact group - in forum $1 \mathrm{P} / \mathrm{N}=7.9$ and in forum $2 \mathrm{P} / \mathrm{N}=3.9$; low-impact group - in forum $1 \mathrm{P} / \mathrm{N}=12.5$ and in forum $2 \mathrm{P} / \mathrm{N}=$ 13.3); therefore, the study confirmed the Meta Learning model, indicating the importance of expansive emotional space (Losada Line range) for positive group dynamics.

g) Non-correlation between final evaluation score of course participants and impact on participants' performance, and importance of context for impact also corroborate some results found in previous studies on Organizational Psychology about the impact of training in in-person modality (Abadd, 2000).

In summary, the importance of the variables context, teacher and group were identified for course effectiveness. Relevance of certain characteristics of students' and teachers" "speeches" was also confirmed to generate conversational dynamics that result in higher course effectiveness.

It is also worth stressing some aspects of these results. First, the influence of context. In DL the student is spatially distant and has a more flexible time to study; however, institutional support for such process is decisive. Such support should be manifested in many aspects, for example: when the course is corporate - encouraging coordinators and colleagues, application of learnt content valued by the institution, support materials and technology, etc.; when the course is focused on a general audience - support infrastructure in places where students live; hence the importance of DL poles.

A second element to be stressed is the teacher's importance as a mediator of learning processes. This issue has a strong impact in DL models that are being practiced in Brazil and worldwide. In general, there are three major trends in structuring the teacher's role in the main DL systems:

a) The teacher as a course coordinator, guiding a group of tutors to interact with students; in this case, tutors (usually recently graduated students or 
undergraduate students) are responsible for establishing a direct relationship with students;

b) The teacher-tutor, who coordinates and directly interacts with their students (similar to the situation of in-person courses; this model was adopted in the course under investigation);

c) Course without interactions with teachers or tutors; such courses have a strong self-institutional component, with no human interaction, in which students are guided by didactic materials.

DL is always facing the challenge of combining what is particular and universal in formation. It is known that, to a certain extent, this is a dilemma in general education. A universal curriculum needs to be related to varied particular forms of knowledge, constituents of different identities. Particularly in DL there are specificities: on one hand, heterogeneity of the audience to be served - DL is usually a modality used in extensive geographical areas and large populations; on the other hand, the needs of a certain standardization of learning instruments to make system management feasible. This makes the educational system even more complex and reinforces the challenge.

Such issue can be faced by flexibility of the course design, to match curricular content with students' previous culture and knowledge. In this sense, a secret to achieve effectiveness in this process lies in the teacher's activity, as shown in this study.

This study represents the first steps in the development of a possible path to evaluate effectiveness of distance courses using the Meta Learning model adapted to the virtual learning environment. A methodology that aims to reflect dynamics - which are not linear - of interactive processes was proposed, a methodology that sees learning as a non-deterministic process (cause-effect), but as a complex phenomenon.

\section{References}

[1] Vygotsky, L.S.: A formação social da mente: O desenvolvimento dos processos psicológicos superiores. Martins Fontes, São Paulo (1998) [Trad. José Cipolla Neto et alli]

[2] Vygotsky, L.S.: Psicologia pedagógica. Martins Fontes, São Paulo (2004) [Trad. Paulo Bezerra]

[3] Vygotsky, L.S.: Pensamento e linguagem. Martins Fontes, São Paulo (2005) [Trad. Jefferson Luiz Camargo; Rev. José Cipolla Neto]

[4] Abbad, G.: Um modelo integrado de avaliação do impacto do treinamento no trabalho IMPACT. Tese de Doutorado. Universidade de Brasília, Brasília (2000)

[5] Losada, M.: Meta Learning. Conferência ministrada no curso Art of Business Coaching. Brasília, Manuscrito (2000)

[6] Losada, M., Heaphy, E.: The role of positivity and connectivity in the performance of business teams: a nonlinear dynamics model. In: Cameron, K.S., Caza, A. (eds.) Contributions to positive organizational scholarship, vol. 47(6), pp. 740-765. American Behavioral Scientist (2004) 